at the 3'-terminus of some RNA viruses is evidence against their antiquity, not for it, as Maizels and Weiner write ${ }^{1.5}$.

Further, the poly $(\mathrm{C})$-forming activity of self-splicing introns cannot be viewed as a vestige of "an early RNA replicase" . Drift would have destroyed this activity immediately after it ceased to be functional were it not intrinsically associated with splicing chemistry. And if poly(C)forming activity is intrinsically associated with splicing, it would be found in selfsplicing introns whenever they emerged.

We are agnostics concerning the interesting 'genomic tag' model. But it is weak because it takes an isolated aspect of viral biochemistry and transforms it into a model for the origin of translation in a distant RNA world without a supporting evolutionary tree to place this trait in the progenote (the most recent common ancestor of modern organisms). Moreover, it is quite likely that the trait is adaptive and therefore could have arisen in the modern world, as Maizels and Weiner have acknowledged elsewhere ${ }^{5}$.

Maizels and Weiner's disagreement with our focus on a 'breakthrough organism' is less than clear. In the context of the model, there must have been a first organism to contain a genetically encoded message which (given the homology of all modern ribosomes) lived before the progenote. This does not mean, nor have we written, that translation arose "all at once", or that translating and nontranslating organisms did not coexist for an arbitrary time. But such a major metabolic innovation must have caused extinctions in a metabolically and ecologically complex RNA world. This means that the breakthrough organism is (at best) the most ancient organism whose metabolism can be reconstructed simply by extrapolation from the biochemistry of modern organisms. Thus, models of the organism, together with prebiotic chemistry, must be the starting points for speculation about the RNA world.

A study of the chemical details of modern metabolism allows us to build these models in some detail ${ }^{2.6}$. For example, deoxyribonucleotides are biosynthesized from ribonucleotides, suggesting that RNA evolved before DNA. However, three (or four) mechanistically distinct ribonucleotide reductases exist in the modern world ${ }^{7}$. In the model, this implies that the breakthrough organism used DNA to store genetic information". This is yet another indication of the metabolic complexity of the last ribo-organism. STEVEN A. BENNER

Laboratory for Organic Chemistry, Swiss Federal Institute of Technology, Zurich, Switzerland

Andrew D. Ellington Department of Molecular Biology,

Massachusetts General Hospital,

Boston, Massachusetts 02114, USA

1. Maizels, N. \& Weiner, A.M. Nature 330, 616 (1987).

Benner, S.A. et al. Cold Spring Harb. Symp. quant. Biol. 52. (1988).

White, H.B. III J molec, Evol, 7, 101-104 (1976).

Visser, C.M. \& Kellogg, R.M. J. molec. Evol. 11, 171 (1978)

Weiner, A.M. \& Maizels, N. Proc. nutn. Acad. Sci. 84, 738 (1987)

6. Benner, S.A. \& Ellington, A.D. Nature 329.295 (1987)

Hogenkamp, H.P.C., Follmann, H. \& Thauer, R.K. FEBS Lett. 219, 197-201 (1987)

8. Benner, S.A. Redesigning Life 115 (Springer, Berlin, 1988)

\title{
Potential of ferroelectrics as radiation shields
}

SiR-In the commentary on the radiation hazards of space missions (Nature 330, $709-710 ; 1987)$ Letaw et al. conclude that a $30-\mathrm{cm}$-thick aluminium shield reduces the dose by about $40 \%$ at solar minimum and by less than $25 \%$ at solar maximum. These estimates are obviously based on the well-known processes of loss of energy of charged particles in matter, which do not depend on the dynamics of solid state. But there is one solid-state effect with the potential of providing effective shielding without increasing the mass of the absorber.

In the case of ferroelectric targets of barium titanate and triglycine sulphate at their Curie temperatures, we have observed ${ }^{\prime}$ a $2-3 \%$ increase in the energy loss of electrons of 620 and $942 \mathrm{keV}$. In their theoretical estimate of this anomalous energy loss at the Curie temperature, Ginsberg and Sobyanin obtained a value several times smaller but predicted a much larger loss in the case of the ferroelectric substance tris-sarcosine calcium chloride? Ayyub et al. observed about $12 \%$ increase in energy loss in one of their triglycine sulphate samples for $624-\mathrm{keV}$ electrons ${ }^{3}$, and we observe an almost $100 \%$ increase for $1.7-\mathrm{MeV}$ electrons in barium titanate.

This anomalous increase in energy loss of charged particles in ferroelectrics at the Curie temperature is expected to become more pronounced at relativistic energies. Were this found to be so, ferroelectrics would have many advantages as a shielding material in space missions. There are many ferroelectrics with convenient Curie temperatures. Many of them have high hydrogen content and so can efficiently slow down fast neutrons, and some of them contain elements of high atomic number, which would be efficient gamma attenuators. Hence this process deserves to be investigated in greater detail.

\section{Department of Physics, Gulbarga}

M.T. Lagare University,

Gulbarga, 585 106, India

N. UMAKANTHA

Department of Physics, Karnatak University,

Dharwad, 580003 , India

Lagare, M.T. \& Umakantha. N. Phys. Rev. Lett. 49.1431 1433 (1982).

Ginsberg, V.L. \& Sobyanin, A.A. Soviet solid state Phys. 25, 1163-1164 (1983).

Ayyub. P. et al. Phys. Rev. B32. 2835-2840 (1985).

\section{Size of the Sun in the seventeenth century}

SIR-Our report' that the solar diameter increased during the second half of the seventeenth century was recently criticised $^{2}$ on the basis of two assumptions concerning the accuracy of the seventeenthcentury observations made by Picard and his colleagues: that Picard had observed the polar diameter of Jupiter; and that the accuracy of the micrometer method developed by Auzout could not have enabled the detection of a 3 arc s variation of the diameters of the Sun and planets.

There is no evidence to substantiate such assertions, however. In his original manuscripts ${ }^{3}$, Picard did not state explicitly which diameter he was measuring, except in the case of the Sun and Saturn, where both the horizontal and vertical diameters were measured. In Picard's time, however, the usual procedure was to measure the horizontal diameter of a planet close to meridian transit, when it can best be seen and when refraction effects are minimal. The horizontal diameter would then be close to the equatorial diameter. Picard compiled elaborate tables of atmospheric refraction to deal with that problem, and measurements of equatorial diameter by Newton and Pound ${ }^{+}$are in good agreement with Picard's results.

In relation to the accuracy of the filar micrometer method, Cassini measured the ellipticity of Jupiter in 1691 . He found an oblateness of $2.7 \mathrm{arcs}$, which is close to the real value ( 3 arc s). It should be noted that Picard and Cassini used the same equipment. Their refractor was fitted with a filar micrometer. The micrometer screw was carefully calibrated by measuring the distance between nine hairs placed in the eyepiece. Thus, they could achieve an accuracy of 1 arc $s$ on the apparent diameters of the Sun and planets". This was discussed at length by Auzout in his treaty "Manière exacte" in 1667 (ref. 6). The document mentioned by O'Dell and Van Helden was a preliminary report to the Royal Society in December 1666 (ref. 7).

It seems likely, therefore, that it was the equatorial diameter of Jupiter that Picard measured. Comparing his observations only with polar diameters raises serious problems. The difference between Picard's observations and the actual equatorial diameters is only $1.3 \pm 0.7$ arc $\mathrm{s}$. This discrepancy arises mainly from eight (out of eighteen) observations of Jupiter, made close to opposition. The difference can be explained by the "irradiation" effect: the contrast of a bright object, Jupiter, against a dark sky (near opposition) would result in an overestimation of the diameter. The irradiation effect was well known at the time of Picard, and because of this effect, most observers made their observations when Jupiter was in quadrature, $90^{\circ}$ from the Sun-Earth 